\title{
Adverse effects of paternal chemotherapy exposure on the progeny brain: intergenerational chemobrain
}

\author{
Anna Kovalchuk ${ }^{1,2}$, Yaroslav Ilnytskyy², Rafal Woycicki², Rocio Rodriguez-Juarez ${ }^{2}$, \\ Gerlinde A.S. Metz ${ }^{1,3}$ and Olga Kovalchuk ${ }^{2,3}$ \\ ${ }^{1}$ Canadian Center for Behavioural Neuroscience, Department of Neuroscience, University of Lethbridge, Lethbridge, AB, \\ T1K3M4, Canada \\ ${ }^{2}$ Department of Biology, University of Lethbridge, Lethbridge, AB, T1K 3M4, Canada \\ ${ }^{3}$ Alberta Epigenetics Network, Calgary, AB, T2L 2A6, Canada \\ Correspondence to: Gerlinde A.S. Metz, email: gerlinde.metz@uleth.ca \\ Olga Kovalchuk, email: olga.kovalchuk@uleth.ca
}

Keywords: chemotherapy, brain, transcriptome, epigenetics, transgeneration effects

Received: January 18, $2016 \quad$ Accepted: April 27, $2017 \quad$ Published: January 23, 2018

Copyright: Kovalchuk et al. This is an open-access article distributed under the terms of the Creative Commons Attribution License 3.0 (CC BY 3.0), which permits unrestricted use, distribution, and reproduction in any medium, provided the original author and source are credited.

\section{ABSTRACT}

Recent advances in cancer treatments have led to significant increases in cure rates. Most cancer patients are treated with various cytotoxic chemotherapy regimens. These treatment modalities are mutagenic and genotoxic and cause a wide array of late-occurring health problems, and even exert a deleterious influence on future offspring. The adverse effects from exposed parents on offspring are referred to as transgenerational effects, and currently little is known about chemotherapy-induced transgenerational effects. Furthermore, transgenerational effects have not been studied in the brains of progeny of exposed parents. In this study, we analyzed the existence and molecular nature of transgenerational effects in the brains of progeny of animals exposed to three common chemotherapy agents: cyclophosphamide (CPP), procarbazine (PCB) and mitomycin C (MMC). For the first time, our results show that paternal exposure to chemotherapy drugs causes transgenerational changes in the brain of unexposed progeny. Although no DNA damage was observed in terms of $\gamma \mathrm{H} 2 \mathrm{AX}$ levels, some alterations were found in levels of PCNA, protein involved in DNA repair, replication and profileration. Furthermore, there were changes in proliferation and apoptosis proteins BCL2 and AKT1, the proteins associated with DNA methylation, DNMT1 and MeCP2. Some altered expression trends were noted in proteins involved in myelin biogenesis, MBP and MYT1L. Moreover, global transcriptome profiling revealed changes in over $\mathbf{2 0 0}$ genes in the whole brains of progeny of animals exposed to CPP, and the changes in the levels of FOXP2 and ELK1proteins were confirmed by western blot analysis. These findings suggest that paternal chemotherapy significantly affects offspring brain development and may affect brain functioning. This research provides a key roadmap for future investigations of the novel phenomenon of transgenerational effects of chemotherapy in the brain of progeny of exposed parents.

\section{INTRODUCTION}

Cancer is the leading cause of death in Canada. In addition, in Canada today, cancer is the leading cause of death in children and young adults, and currently one in 400 Canadian adults are survivors of childhood cancer $[1,2]$. The number of new cases in Alberta alone is expected to double to more than 26,000 cases per year by 2025 [3]. In recent decades, advances in treatment have led to significant increases in cancer survival and cure rates. In the United States, the five-year survival rate (1985-1999) for children diagnosed with cancer was $75.8 \%$ [4]. For Americans aged 20 to 39, the prevalence of childhood cancer survivors is approximately 1 in 640 [5]. 
Most cancer patients are treated with various cytotoxic chemotherapy regimens $[6,7]$. These treatment modalities are mutagenic and genotoxic; therefore, more than twothirds of cancer survivors experience a wide array of lateoccurring health problems $[1,2,6-8]$.

Additionally, for cancer patients, their ability to have healthy children is of great importance, especially due to the fact that it is now well-accepted that parental exposure to various environmental stressors can exert negative influences on future offspring. These adverse effects in the offspring of exposed parents are referred to as transgenerational or intergenerational effects $[9,10]$.

There is a wealth of evidence that radiation exposure effects can span several generations, and leads to transgenerational effects in the offspring of exposed parents [11-13]. While transgenerational effects have been reported in exposed human populations including atomic bomb survivors, individuals affected by the Chernobyl accident, and those living near nuclear test sites [14], their nature and magnitude in the exposed human population remains controversial $[15,16]$. Nevertheless, they are also welldocumented in rodents, and rodent models are accepted as valuable, albeit not ideal, tools for transgenerational effect analysis, especially for transgenerational carcinogenesis [11, 17-19]. Furthermore, the observed radiation-induced transgeneration effects are primarily paternal in nature $[11,20]$, with irradiation manifesting both phenotypically and at the genome level in affected progeny. Phenotypic effects include decreased fertility, teratogenic effects and an increased predisposition to cancer [18, 21, 22]. Genome alterations in the progeny of exposed parents appear as increased levels of DNA damage and mutation rates, as well as elevated frequencies of chromosome aberrations and micronuclei [11, 18, 23]. Furthermore, progeny of exposed parents also exhibit altered gene expression patterns and epigenetic changes [24, 25].

While the initial data on transgenerational effects have been obtained from analysis of the progeny of radiation-exposed parents, further studies have revealed that a wide variety of other genotoxic agents induce transgenerational effects $[6,17,26]$, such as pesticides and air pollution [27]. Also, there is strong evidence that parental exposure to anticancer chemotherapy drugs can cause heritable transgenerational effects $[6,7]$. The pioneering works from the Dubrova laboratory revealed that paternal exposure to clinically relevant doses of bleomycin, cyclophosphamide, and mitomycin $\mathrm{C}$ lead to statistically significant and dose-dependent increases in mutation rates in the germline of treated male mice and caused transgenerational genome instability in the offspring of exposed animals [6, 7].

While it has been established that transgenerational effects exist, the exact molecular mechanisms of these phenomena have yet to be defined. Furthermore, recent evidence suggests that transgenerational effects may be epigenetic in nature $[10,18,28]$ and are associated with gene expression changes. In addition, the existence of transgenerational effects has been established in organs that are known targets of blood malignancies, such as the spleen, thymus and liver, as well as the white blood cells [24]. This is primarily due to the fact that transgenerational radiation effects often manifest themselves as an increased predisposition to blood cancer. Furthermore, several transgenerational effects of social and psychological stressors have been shown to affect the brain of their offspring [29, 30]. Nevertheless, currently nothing is known about the effects of paternal exposure to chemotherapy drugs on the brain of their unexposed offspring.

Here we set out to analyze molecular gene expression signatures and protein expression profiles in the brains of offspring from males that were exposed to three widely-used cytotoxic chemotherapy drugs, cyclophosphamide (CPP), procarbazine (PCB) and mitomycin $\mathrm{C}$ (MCC), and prior to conception. We hypothesized that paternal exposure to these cytotoxic drugs may affect gene expression and protein levels in the brain of unexposed offspring.

\section{RESULTS AND DISCUSSION}

\section{Paternal chemotherapy altered levels of KU70 and PCNA in the progeny}

Histone $\mathrm{H} 2 \mathrm{AX}$ is rapidly phosphorylated at Ser139 upon induction of DNA strand breaks, and it can be effectively detected using specific antibodies [31]. Analysis of histone H2AX phosphorylation (levels of $\gamma \mathrm{H} 2 \mathrm{AX})$ is widely used to assess the extent of damage to cellular DNA [31]. The induction of DNA damage and H2AX phosphorylation constitute well-established transgenerational effects. Therefore, the levels of $\gamma \mathrm{H} 2 \mathrm{AX}$ in frontal cortex (FC) and brain (BR) tissues of the progeny of control and exposed animals were analyzed.

Surprisingly, no H2AX phosphorylation was detected in any tissue in any of the three independent iterations of the experiment (data not shown). This may indicate that there was no DNA damage induced in the brain tissues of progeny by the pre-conception paternal exposure to chemotherapy agents. Moreover, the brain is highly plastic and has a huge capacity to repair DNA damage and maintain DNA integrity. Additional studies would be needed to analyze the levels of DNA damage in the brain of progeny of exposed parents using other methods.

Analysis of p53, a key cellular gatekeeper involved in orchestrating DNA damage responses, cell cycle control, and apoptosis [32, 33], did not reveal any significant changes in its levels in either FC or BR samples (Figure 1). No significant changes were seen in the levels of KU70, a DNA repair protein which participates in the non-homologous end-joining mechanism of DNA 
repair [34], albeit some insignificant trends were noted (Figure 1).

Contrarily, the level of another protein, the proliferating cells nuclear antigen (PCNA) involved in DNA replication and repair DNA synthesis [34, 35], was statistically significantly $(p<0.05)$ substantially reduced in all frontal cortex samples in response to all treatments, and in the whole brain samples in response paternal MMC and PCB exposures (Figure 1). The observed decrease in the levels of PCNA may indicate alterations in the levels of cellular growth and proliferation.

\section{Paternal chemotherapy changed levels of BCL2 and AKT1 in the progeny}

DNA damage and repair are closely linked to the control of the cell cycle and apoptosis. To repair DNA damage, cells need time, which is gained by implementing cell cycle arrest. In the case of inefficient or delayed repair of DNA damage, cells undergo apoptosis.

Although no direct evidence of DNA damage in the brain tissues of progeny of exposed parents was observed, this study proceeded to analyze the levels of several key proteins involved in cell cycle control and apoptosis. Among these, the levels of cellular anti-apoptotic protein BCL2 [36] was analyzed. A significant decrease in BCL2 levels was found in the $\mathrm{FC}$ of progeny of animals exposed to all MMC and PCB chemotherapy drugs (Figure 1). A decrease in the levels of anti-apoptotic BCL2 may lead to elevated levels of apoptosis in the FC of progeny. Moreover, FC samples also exhibited reduced levels of PCNA.

In the BR, contrarily, paternal exposure to MMC led to an increase in BCL2 levels (Figure 1). Such an increase may result in a reduction in the levels of apoptosis in brain regions other than the FC. In the future, it would be important to analyze the levels of other apoptosis-related proteins in the brains of progeny of chemotherapy-exposed parents. It would also be prudent to conduct staining to reveal apoptotic cells in order to determine if the levels of apoptosis were indeed affected and, if so, in which brain region cell types this occurred.

Also noted was a decrease in the levels of AKT1 in the FC of progeny of fathers exposed to CPP and MMC, and in the whole brains (BRs) of progeny of PCB-exposed fathers (Figure 1). AKT1 is important in controlling organismal growth and development, and AKT1-deficient mice display impaired overall growth [37]. Moreover, AKT1 plays a key part in brain development and neuronal survival $[37,38]$. Therefore, a reduction in AKT1 levels may be a concern that deserves future attention, especially when it is parallel with a reduction in PCNA levels and decreased levels of anti-apoptotic BCL2 proteins. A reduction of AKT1 levels in very young animals may negatively affect brain growth and development and may, therefore, have detrimental consequences for progeny of chemotherapy-exposed parents. More studies would be needed in the future to understand the roles of AKT1 and AKT1-associated signaling in transgenerational changes in the brain.

\section{Exposure to MMC and PCB Affect DNMT1 and MeCP2 levels}

Gene expression is controlled by DNA methylation. DNA methyltransferases regulate methylation patterns in the genome. DNMT1 functions as a maintenance methyltransferase and is responsible for maintaining the methylation patterns upon replication [39]. Changes in its expression may, in turn, influence DNA methylation levels. The DNMT1 level was found to be significantly reduced in the FC samples of progeny of CPP- and MMCexposed fathers $(p<0.05)$ (Figure 2$)$. No significant changes were seen in the BR samples. Similar trends were found in the levels of the methyl-CpG binding protein, MeCP2 (Figure 2), which binds to methylated DNA in order to stabilize DNMT1 functions and induce chromatin remodeling and gene silencing [39]. The loss of MeCP2 may be associated with genome instability. Furthermore, previous studies have shown a decrease in the levels of $\mathrm{MeCP} 2$ in progeny of radiation-exposed parents [24]. Additionally, MeCP2 is critical for establishing and maintaining neuronal networks and brain anatomy $[40,41]$. Therefore, more studies are needed to dissect the biological and functional repercussions of the observed loss of MeCP2 levels in the brains of progeny of chemotherapy-exposed parents.

\section{Myelin transcription factor MYT1L and myelin basic protein levels change in progeny of animals exposed to MMC and PCB}

Maternal stress can influence proteins involved in the etiology and pathogenesis of neurological diseases [42]. With this in mind, the levels of myelin basic protein (MBP) and the myelin transcription factor, MYT1L, in the brains of progeny of chemotherapy-exposed parents were analyzed. MYT1L is involved in regulating the development of the nervous system $[43,44]$. It is one of three factors that are sufficient to convert mouse embryonic and postnatal fibroblasts into functional neurons. MBP is important in the process of myelination of the nerves in the nervous system [45].

It was noted that paternal exposure to $\mathrm{MMC}$ resulted in decreased levels of in the FC of unexposed progeny of chemotherapy-exposed parents, whereas paternal exposure to PCB resulted in decrease of MYT1L in the BR samples of progeny (Figure 3). No difference was observed in response to paternal CPP exposure. Recent studies have suggested that MYT1L may be a candidate gene for intellectual disability (ID) [44]. Therefore, in the future, it would be important to further analyze the levels 
of MYT1L and its role in the brain of progeny of exposed parents. MBP levels need further investigation, since the current protein level trends are inconclusive.

In general, very little is known about the effects of MCC and PCB on the nervous system of exposed animals as well as on the nervous system of progeny of exposed animals. In contrast, there is a substantial body of evidence about the effects of CPP. Transient hippocampal-based memory deficits following a single injection of CPP were found in two independent mouse model-based studies [46, 47]. Also, CPP-treated rats showed significantly impaired behavior, namely poor performance on the novel place recognition task and the contextual fear conditioning task, which was likely due to a disruption
FRONTAL CORTEX p53

KU70

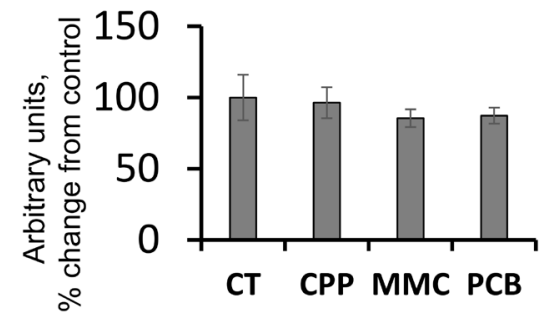

PCNA
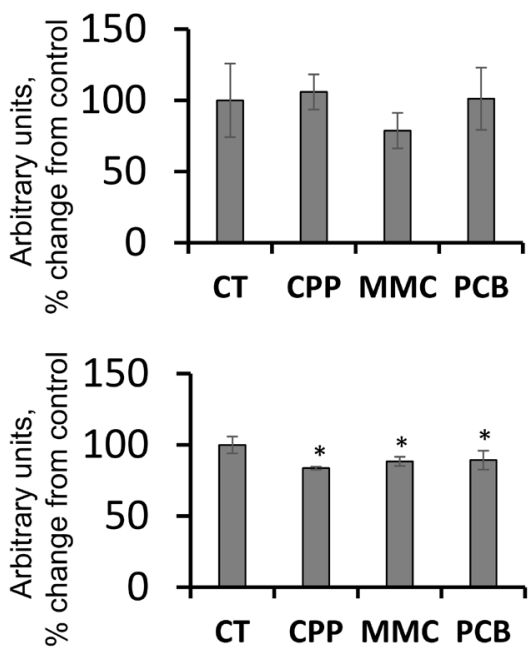

AKT1

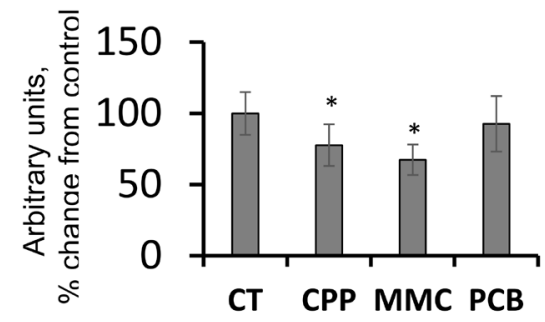

BCL2
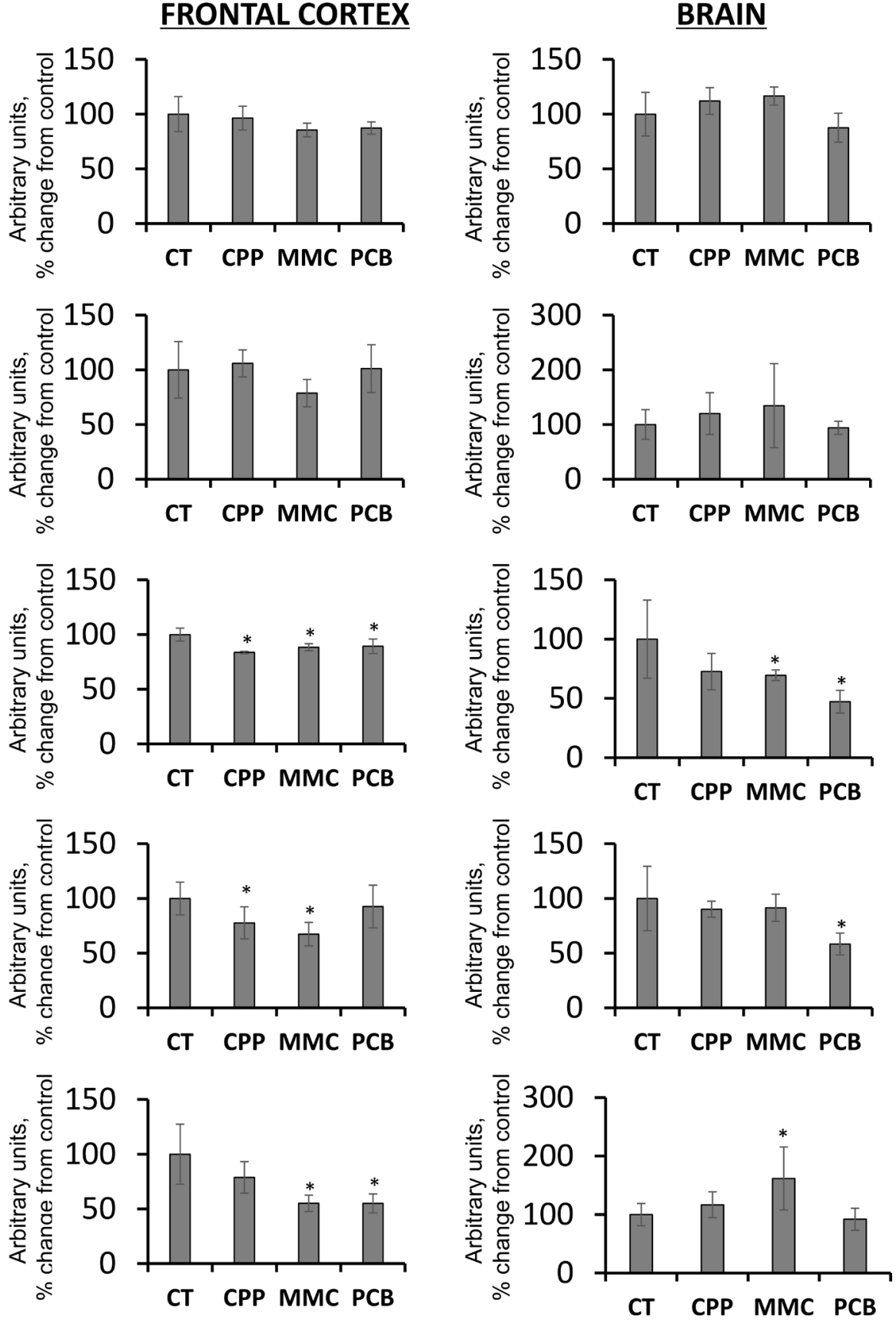

Figure 1: Changes in the levels of DNA damage-, DNA repair- and apoptosis-related proteins in the frontal cortex (FC) and whole brain (BR) tissues of progeny of chemotherapy-exposed parents. $\mathrm{CPP}$ - cyclophosphamide; $\mathrm{MMC}$ - mitomycin $\mathrm{C}$; PCB - procarbazine. Data are shown as average (with SD) arbitrary units of intensity calculated from six individual data points per each experimental group. Due to protein size differences and scarcity of tissue, membranes were re-used several times. Each data point was related to an average intensity of actin samples in a corresponding group and expressed as percent change from controls. sterisks $\left(^{*}\right)$ indicate statistically significant $(p<0.05)$ difference to unexposed control Student's $t$-test. 
in the hippocampal-based memory function [48]. These CPP-treated animals also had a significant reduction in the number of mature neurons, suggesting impaired neurogenesis.

Furthermore, Hsu et al. (1987) showed that paternal CPP treatment resulted in significant changes in several neurotransmitter enzymes in various brain regions of the immediate progeny [49]. Also, the F1 progeny of male rats chronically exposed to CPP exhibited behavior deficits when compared to controls [50, 51]. At that time, the authors believed that this was likely due to genetic effects induced by CPP exposure.

In contrast, the results of Lyons et al. (2011) indicate that CPP acutely reduces the survival of newlyborn hippocampal cells, but does not have any longterm effects on spatial working memory or hippocampal proliferation [52]. Examination of the offspring of treated animals showed an increased post-natal mortality rate, paralleled by diminished learning capacity and reduced spontaneous activity in the adults. These changes also persisted in the second generation of exposed animals. Analyses of the brains of the first and second generations showed diminished activity of hippocampal choline acetyltransferase, as well as a decreased level of norepinephrine in the fronto-parietal cortex in the second generation [52].

\section{Global genome expression analysis revealed substantial changes in the whole brain of the progeny in response to paternal CPP exposure}

To further investigate changes in the gene expression in progeny of animals exposed to chemotherapy drugs, whole transcriptome analysis in the FC and BR samples was performed. After averaging the data for five animals per group, significant differences were only found in the BR samples of CPP-exposed animals. There were 137 upand 131 down-regulated genes. After the $-0.3>\log 2>0.3$ cut-off was implemented, 86 up- and 75 down-regulated genes were found.

\section{Reactome analysis}

The information about enriched pathways for reactome analysis was found for 33 up- and 34 downregulated genes. The analysis showed enrichment in upregulated genes belonging to "disease", "gene expression" and "metabolism" reactomes, and in down-regulated genes belonging to "metabolism", "cell cycle", "developmental

FRONTAL CORTEX

BRAIN
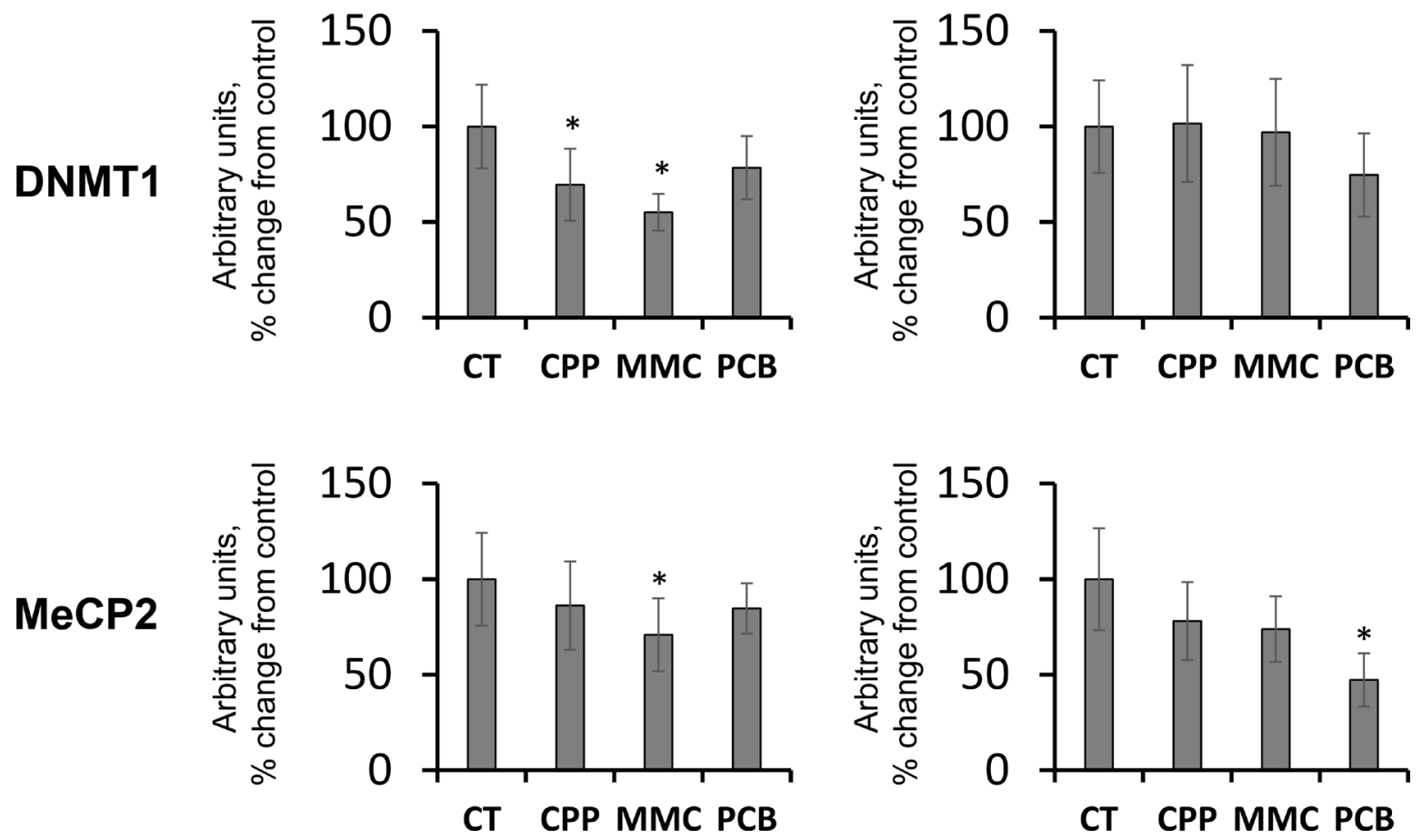

Figure 2: Changes in the levels of DNMT1 and MeCP2 in the brain tissues of progeny of chemotherapy-exposed parents. CPP - cyclophosphamide; MMC - mitomycin C; PCB - procarbazine. Data are shown as average (with SD) arbitrary units of intensity calculated from six individual data points per each experimental group. Due to protein size differences and scarcity of tissue, membranes were re-used several times. Each data point was related to an average intensity of actin samples in a corresponding group and expressed as percent change from controls. Asterisks $\left(^{*}\right)$ indicate statistically significant $(p<0.05)$ difference to unexposed control, Student's $t$-test. 
biology" and "gene expression" reactomes (Figure 4A). Genes belonging to "disease", "metabolism" and "gene expression" reactomes were common in the up- rather than the down-regulated gene group. In contrast, genes belonging to "cell cycle" and "chromatin organization" reactomes were more common in the down-regulated gene group.

\section{Gene ontology analysis}

Next, functional annotation analysis was performed using DAVID's Functional Annotation Chart, with GO terms found for approximately $60 \%$ of all genes in the upand down-regulated categories of genes. In the "biological processes" category, for up-regulated genes, enrichment of over two-fold was found for GO terms related to protein metabolism, cell-cell signaling, cell adhesion and transport (Figure 4B), whereas for down-regulated genes, it was for DNA and RNA metabolism, cell-cell signaling, cell cycle and proliferation, cell death, cell organization and biogenesis and several others. A comparison of the up- and down-regulated groups showed substantial overrepresentation of genes belonging to RNA and DNA metabolism, cell-cell signaling, cell cycle and proliferation and cell death in the down-regulated group as compared to up-regulated genes (Figure 4B).

Analysis of GO terms for "molecular function" for up-regulated genes showed enrichment of over two-fold for kinase activity, whereas in the down-regulated genes for cytoskeletal activity, nucleic acid binding and enzyme regulator activity (Figure 4C), the latter three groups were significantly overrepresented in the down-regulated gene category.

\section{Pathway analysis using KEGG}

Unilateral analysis of differentially expressed genes using KEGG software showed the following pathways to be up-regulated in progeny of CPP-exposed animals: oxidative phosphorylation, Alzheimer's, Parkinson's and Huntington's diseases, and cardiac muscle contractions (Figure 5A). Bilateral analysis showed 20 different KEGG pathways up-regulated, including those mentioned above, as well as nucleotide excision repair, RNA polymerase, ribosome biogenesis, mRNA surveillance, RNA degradation, RNA transport and many others (Figure 5B).

A more detailed picture was obtained for differentially expressed genes in 10 different categories, including Alzheimer's, Parkinson's and Huntington's diseases, neurotrophin signaling pathway, mRNA surveillance pathway, oxidative phosphorylation, nucleotide excision repair, spliceosome, proteasome and ribosome function (Supplementary Figures 1-10). For example, cytochrome complexes I, III, IV and V (Cx I, Cx III, Cx IV, Cx V) were up-regulated, whereas cytochrome complex Cx II and Casp3 were down-regulated in
FRONTAL CORTEX
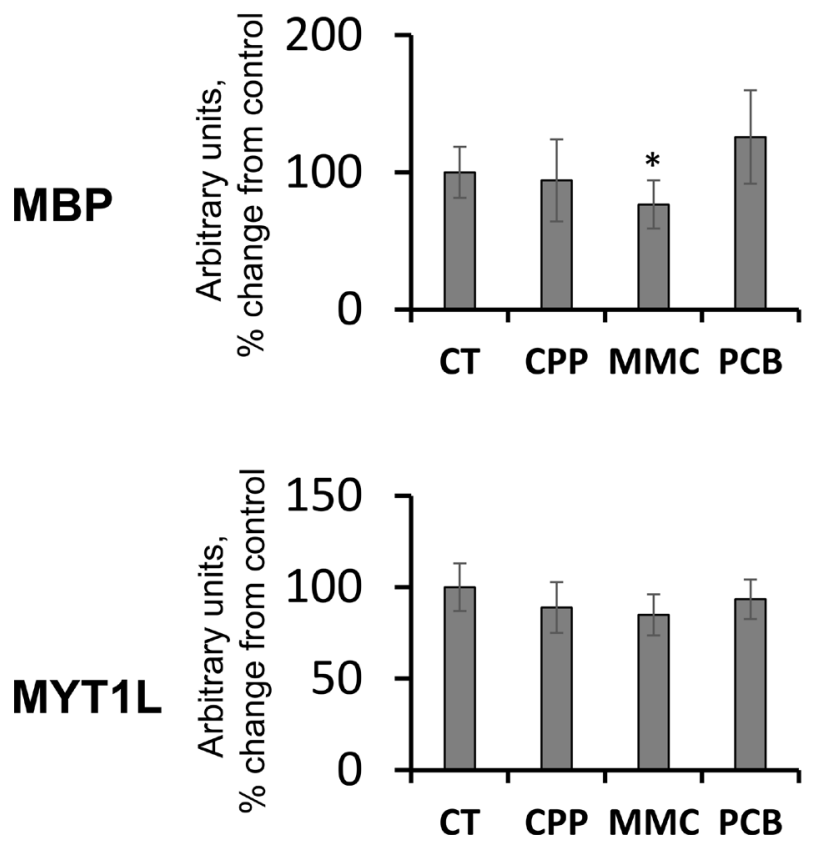

BRAIN
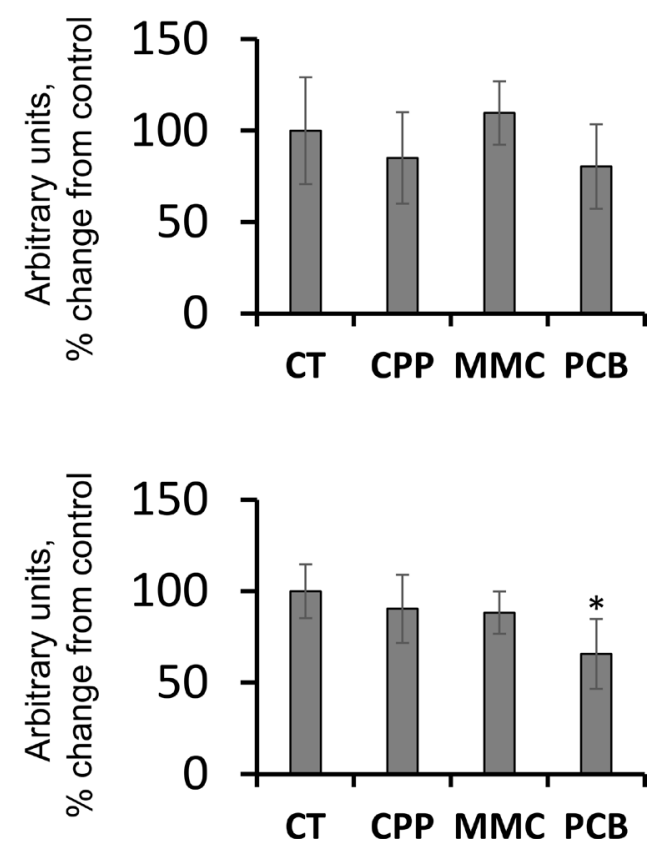

Figure 3: Levels of MBP and MYT1L in the brain tissues of progeny of chemotherapy-exposed parents. CPP cyclophosphamide; MMC - mitomycin C; PCB - procarbazine. Data are shown as average (with SD) arbitrary units of intensity calculated from six individual data points per each experimental group. Due to protein size differences and scarcity of tissue, membranes were re-used several times. Each data point was related to an average intensity of actin samples in a corresponding group and expressed as percent change from controls. Asterisks $\left(^{*}\right)$ indicate statistically significant $(p<0.05)$ difference to unexposed control, Student's $t$-test. 
A

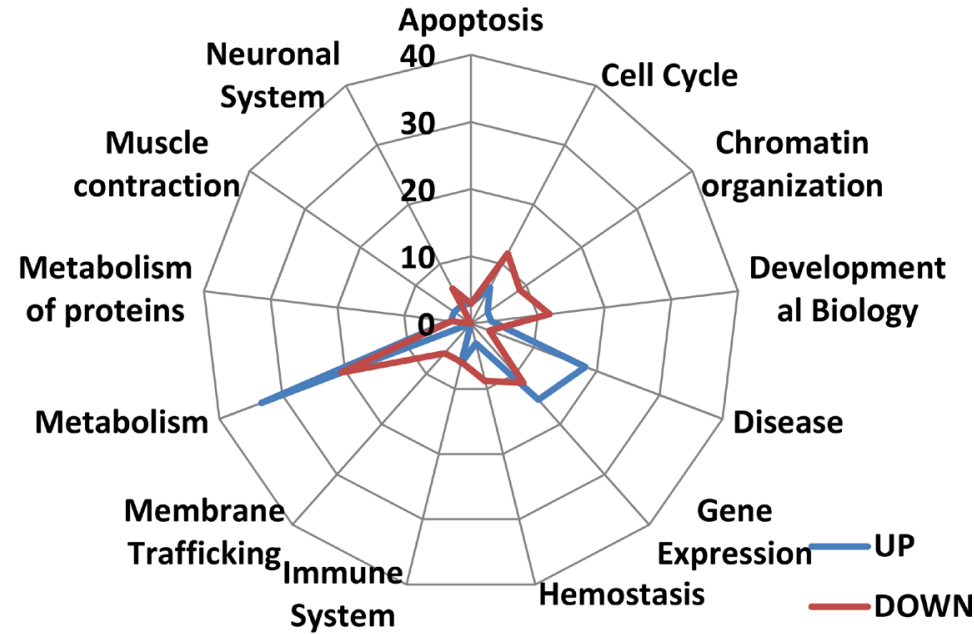

B

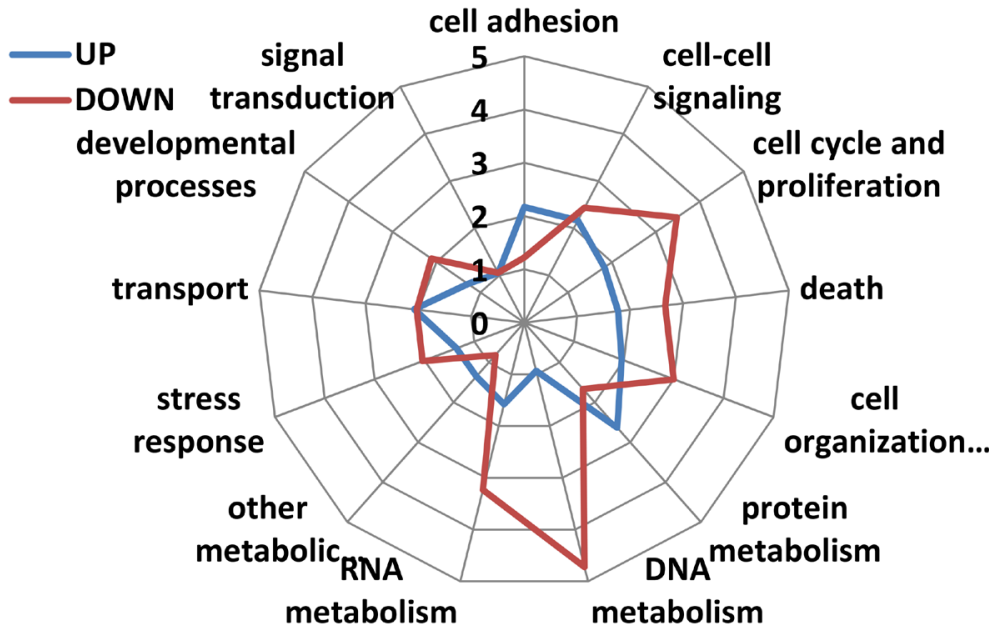

C

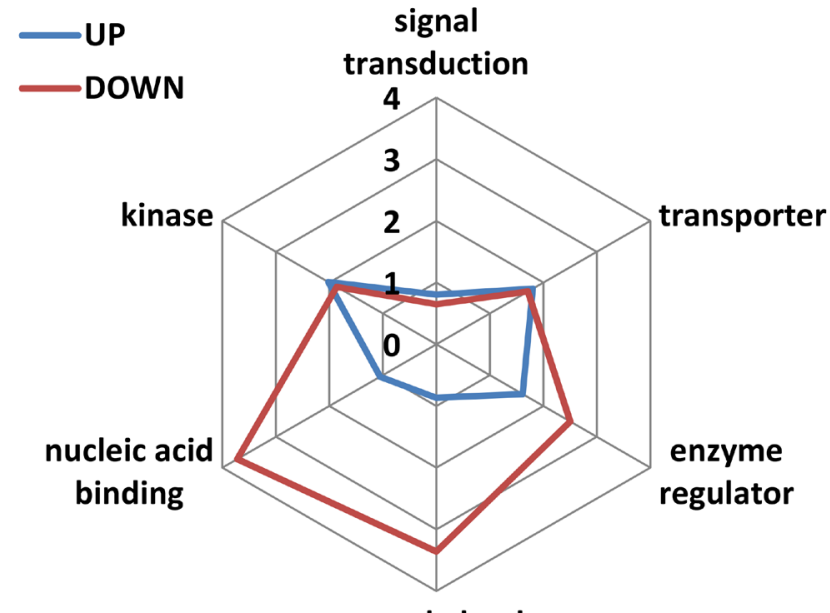

cytoskeletal

Figure 4: Reactome analysis and analysis of GO terms for biological processes and molecular functions. (A) Reactome analysis. Reactome web graphic shows the percentage of up- and down-regulated genes belonging to a particular reactome out of all genes for which reactome data were found. (B) GO terms enrichment analysis for biological processes. Graphic shows the percentage of up- and down-regulated genes belonging to a particular reactome out of all genes for which GO terms for biological processes were found. (C) GO terms enrichment analysis for molecular functions. Graphic shows the percentage of up- and down-regulated genes belonging to a particular reactome out of all genes for which GO terms for molecular functions were found. 
Alzheimer's, Parkinson's and Huntington's diseases (Supplementary Figures 1-3).

The importance of changes in response to CPP was demonstrated by alterations in the neurotrophin signaling pathway (Supplementary Figure 4); progeny of animals exposed to CPP had decreased expression levels of $N R A G E$. NRAGE interacts with the nerve growth factor (NGF) low affinity receptor p75NTR and facilitates cell cycle arrest and NGF-dependent neuronal apoptosis [53]. Expression of calmodulin (CaM) and phosphorylated cAMP-response element binding protein (CREB) decreased in progeny. Disruption of the CaM/CREB pathway could be associated with impaired hippocampal learning and memory [54].

The expression level of several key proteins involved in neurogenesis and neuroprotection were tested further using western blot analysis. Interestingly, the levels of Dcx protein were unchanged in response to CPP (Figure 6). Doublecortin (DCX) is a cytoskeletonassociated protein that is important for neurogenesis, including migration, axonal guidance and dendrite sprouting [55]. While it was down-regulated on the RNA level, its protein levels were unchanged. This difference may be explained by the compensatory involvement small RNAs or changes in RNA stability and the precise mechanisms of this phenomenon need to be studied in the future. Also, protein levels of neuronal transcription factor FOXP2 were slightly, but significantly $(p<0.05)$ upregulated in the progeny exposed to CPP (Figure 6); FOXP2 is important for the development of brain regions involved in speech and language development [56].

In contrast, the levels of ELK1 protein were decreased in the progeny of animals exposed to CPP (Figure 6). ELK1 belongs to the family of transcription factors abundantly expressed in the nervous system. It is known to participate in neuroprotection against toxic stimuli. Neuroprotective role of ELK1 was demonstrated in mouse model of Hungtington's disease [57]. It is possible that progeny of CPP-exposed parents has lower potential of neuroprotection in adverse conditions, although this has to be further demonstrated experimentally.

Several components of oxidative phosphorylation machinery were found to be up-regulated, including
A

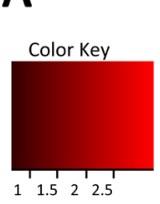

B

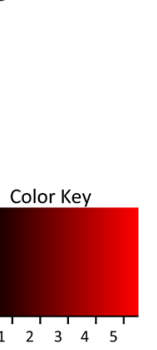

GAGE Up-test: -log10 (p-value)

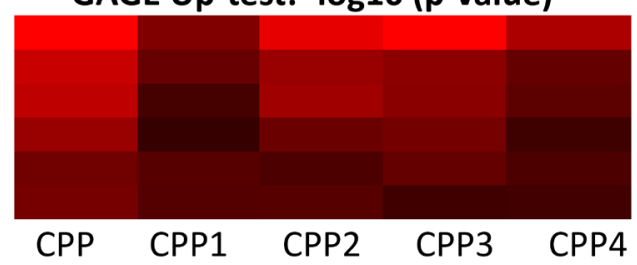

GAGE Two-way test: - $\log 10$ (p-value)

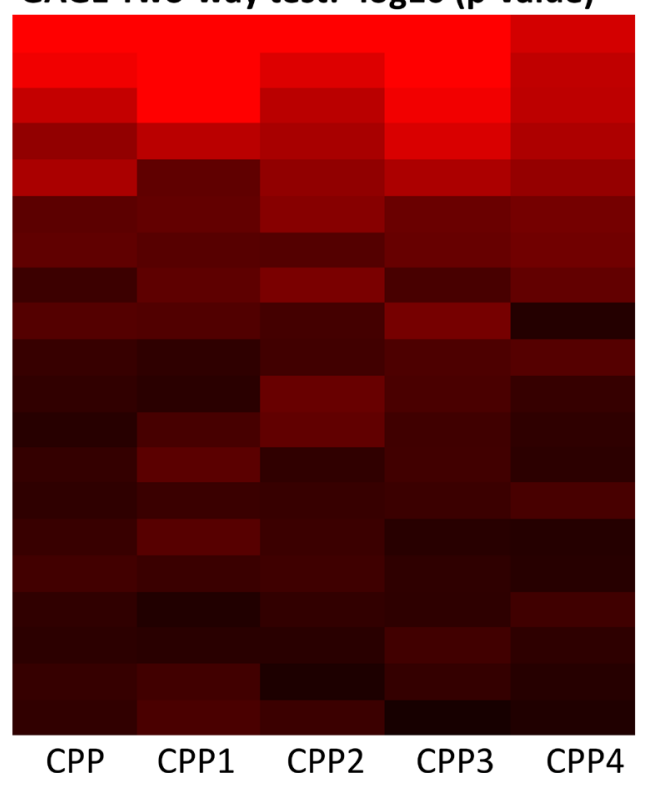

mmu00190 Oxidative phosphorylation

mmu05012 Parkinson's disease

mmu05016 Huntington's disease mmu05010 Alzheimer's disease

mmu04260 Cardiac muscle contraction mmu05150 Staphylococcus aureus infection

mmu03420 Nucleotide excision repair mmu04130 SNARE interactions in vesicular transport mmu03020 RNA polymerase mmu03008 Ribosome biogenesis in eukaryotes mmu04962 Vasopressin-regulated water reabsorption mmu03015 mRNA surveillance pathway mmu03018 RNA degradation mmu00650 Butanoate metabolism mmu04141 Protein processing in ER mmu04120 Ubiquitin mediated proteolysis mmu00020 Citrate cycle (TCA cycle) mmu04260 Cardiac muscle contraction mmu03013 RNA transport mmu03050 Proteasome mmu03040 Spliceosome mmu03010 Ribosome mmu05010 Alzheimer's disease mmu05016 Huntington's disease mmu05012 Parkinson's disease mmu00190 Oxidative phosphorylation

Figure 5: Analysis of pathways deregulated upon parental exposure to CPP. (A) Heat map of changes in various KEGG pathways obtained after unilateral GAGE analysis. Heat maps (red indicates up-regulation) were generated after unilateral analysis using KEGG pathways and feeding the data for gene expression from five animals (labelled as CPP, CPP1, CPP2, CPP3, CPP4 and CPP5), each individually compared to the averaged control. (B) Heat map of changes in various KEGG pathways obtained after bilateral GAGE analysis. Heat maps (red indicates up-regulation) were generated after bilateral analysis using KEGG pathways and feeding the data for gene expression from five animals (labelled as CPP, CPP1, CPP2, CPP3, CPP4 and CPP5), each individually compared to the averaged control. 
components of the electron transport chain, NADH dehydrogenases (Ndufa1, Ndufa2, Ndufa3, Ndufa5, Ndufa 7, Ndufa8), cytochrome C reductases (QCR9, QCR10), and cytochrome C oxidases (COX6A, COX6B, $C O X 7 A, C O X 7 B, C O X 17$ ) (Supplementary Figure 5).

In the nucleotide excision repair pathway, the expression of PCNA was reduced (Supplementary Figure 6). This finding is similar to what was found on the protein level - PCNA protein levels were reduced in the BR samples of progeny of CPP-treated animals. The component in the XPC complex, CETN2, was also downregulated in several animals (Supplementary Figure 6).

In the ribosome biogenesis category, many genes encoding ribosomal proteins were found to be up-regulated, including homologs of L14, L23, L34e, L30e, S12, S15e, S18, S26e, L44e, L27e and L38e (Supplementary Figure 7). Similarly, genes encoding spliceosome Sm proteins were up-regulated in progeny of CPP-exposed animals (Supplementary Figure 8).

Our pioneer study provided the first evidence that paternal exposure to chemotherapy drugs may cause transgenerational changes in the brain of unexposed progeny. Although no DNA damage was observed, changes were found in the levels of DNA repair proteins, cell proliferation and apoptosis, proteins related to DNA methylation, as well as proteins involved in myelin production. All of these proteins are important for brain development and function.
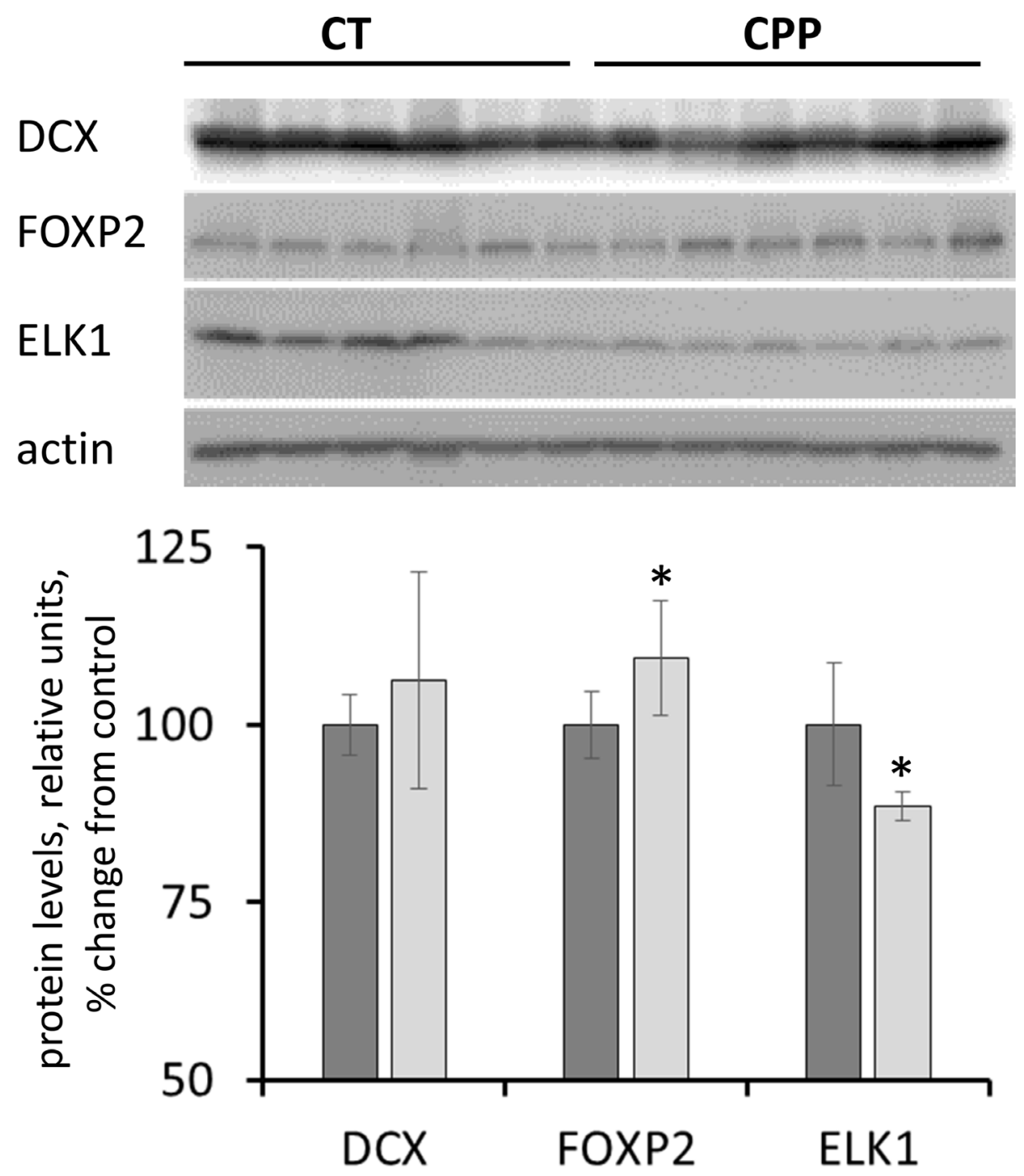

$\square \mathrm{CT} \square \mathrm{CPP}$

Figure 6: Levels of DCX, FOXP2 and ELK1 in whole brain samples of progeny of CPP-exposed parents. Data are shown as average (with SD) arbitrary units of intensity calculated from six individual data points per each experimental group. Due to protein size differences and scarcity of tissue, membranes were re-used several times. Each data point was related to an average intensity of actin samples in a corresponding group and expressed as percent change from controls. Asterisks $\left(^{*}\right)$ indicate statistically significant $(p<0.05)$ difference to unexposed control, Student's $t$-test. 
The present analyses present the first study showing that paternal chemotherapy exposure exerts transgenerational effects in the brain tissues of unexposed progeny, albeit to a moderate extent. Paternal chemotherapy exposure led to altered levels of proteins PCNA, BCL2, AKT1, DNMT1, MeCP2, MBP, MYT1L, FOXP2, and ELK1 in FC and BR tissue, although they were often changed in the opposite direction in these two samples. Transcriptomic analysis showed changes only in response to CPP and only in the BR tissues. Further studies are required to determine why this was the only treatment causing changes in the transcriptome. Furthermore, transgenerational changes may be brainregion specific and this specificity needs to be further analyzed in detail, and, most importantly, using larger animal cohorts

Profound changes in neurotrophin pathway and pathways leading to Alzheimer's, Parkinson's and Huntington's diseases suggest that the progeny of CPPexposed animals may be impaired in various neurological functions and possibly, may even demonstrate altered behavior. Additional molecular, neuroanatomical and behavioral studies are needed to corroborate and extend these pilot results. In the future, it would be important to dissect the cellular changes in the progeny's brain tissue. It would be prudent to correlate protein levels and cellular changes with global gene expression and, most importantly, to analyze small RNA profiles of the brain tissue of progeny of chemotherapy-exposed parents. It would also be interesting to establish region-specificity of the observed changes.

However, in future research, it would be most important to establish whether changes on the molecular level are paralleled by changes in behavior. The decrease in MYT1L levels may lead to altered cognitive functions. Similarly, the loss of AKT1 may affect neuronal development and, thus, influence a myriad of neuronal functions. At the same time, lower levels of ELK1 may result in lower neuroprotective potential and adverse response to stress in the progeny of CPP-exposed parents. Also, altered MeCP2 levels may be associated with altered chromatin, gene expression and brain anatomy.

Here we used several pathway databases (such as $\mathrm{GO}$, Reactome and KEGG) to establish the degree of involvement of different pathways in the molecular changes in the progeny of exposed parents. While these platforms provide excellent tools to analyse biological repercussions of transgenerational effects, they do not allow quantitative pathway activation analysis. This can be achieved using novel platforms such as the OncoFinder or iPANDA. In the future, we will apply OncoFinder and iPANDA to analyse the pathway activation strength $[58,59]$. This approach can also be used to compare transgenerational effects in different organs of the progeny of exposed parents and to correlate gene expression and small RNA expression patterns [60]. Exposures to genotoxic agents can promote and facilitate aging. The observed molecular changes may in turn predispose progeny of exposed parents to accelerated aging. In the future, it would be important to study transgenerational effects in the aging domain [61, 62]. Moreover, in the future it would be important to establish whether changes at the molecular level are parallel by transgenerational changes in behavior and neuroanatomy.

In summation, this study provides a key roadmap for future investigations of the phenomenon of transgenerational effects in the brains of progeny of chemotherapy-exposed parents.

\section{MATERIALS AND METHODS}

\section{Animal model}

In this study, an in vivo murine model ( 8 weeks old male and female BALB/c mice) was used to study the transgenerational effects of chemotherapy drugs. Murine models are widely used, well-characterized and generally accepted for studies of transgenerational effects $[63,64]$. The focus of the analysis was on the molecular changes in the frontal cortex (FC) and whole brain (BR) of the progeny. Animals were housed in a pathogen-free facility and given food and water ad libitum. The study was approved by the University of Lethbridge Animal Welfare Committee according to guidelines developed by the Canadian Council on Animal Care.

\section{Treatments}

\section{Chemotherapy drugs}

Based on previous data [7], transgenerational effects of CPP, PCB and MCC were analyzed. CPP is a widely used anticancer drug and immunosuppressive agent which, following metabolic activation, forms DNA adducts $[6,7]$. Single doses for anticancer treatment in humans are typically between 400 and $1,200 \mathrm{mg} / \mathrm{m} 2$; the equivalent mouse dose for this drug is 130 to $400 \mathrm{mg} / \mathrm{kg}$. Exposing animals to $40-80 \mathrm{mg} / \mathrm{kg}$ of CPP resulted in statistically significant, dose-dependent increases in germline mutations in treated animals [6, 7]. A recommended dose of $150 \mathrm{mg} / \mathrm{kg}$ of CPP was used in this study. PCB is used to treat a number of cancers, including Hodgkin's lymphoma. Metabolites of this drug inhibit DNA polymerase and react directly with DNA causing damage [65]. Human single doses do not exceed a maximum of $150 \mathrm{mg} / \mathrm{m} 2$; the equivalent mouse dose for this drug is a maximum of $50 \mathrm{mg} / \mathrm{kg}[6,7]$. In the same study, paternal exposure to clinically relevant doses of PCB $(50 \mathrm{mg} / \mathrm{kg})$ also led to significantly increased germline mutation rates $[6,7]$. For this research, $13.3 \mathrm{mg} / \mathrm{kg}$ of PCB was used.

The streptomycin-derived antibiotic $\mathrm{MMC}$ is another well-established anticancer drug. It causes DNA damage, and exposure to single doses of $2.5-5.0 \mathrm{mg} / \mathrm{kg}$ 
leads to pronounced increases in germline mutations in mice [6]. Typical single doses to humans are between 10 and $20 \mathrm{mg} / \mathrm{m} 2$, and corresponding mouse doses are 3-6 $\mathrm{mg} / \mathrm{kg}$. To evaluate the transgenerational effects of paternal exposure to $\mathrm{MMC}$, a recommended dose of $5 \mathrm{mg} / \mathrm{kg}$ [6] was chosen.

\section{Husbandry}

Four male BALB/c mice were exposed at eight weeks of age to PCB, CPP or MMC. Eight weeks after exposure, the chemotherapy-treated males were mated with untreated females ( 2 females/1 male). Based on the timing of mouse spermatogenesis, such mating would reveal spermatogonial effects. The litter size in MMC treatment groups appeared to be smaller than others (only 9 pups). Other groups had over 20 pups. Eight pups/group were used in the analysis. The progeny were obtained and sacrificed at the age of one week. To avoid data bias, animals from different litters were evenly distributed among four treatment groups. Upon sacrificing, the brains were dissected to separate the FC from the rest of the brain. Due to the small size of the brains, further dissections were not possible.

The FC and the rest of the brain (BR) were frozen and stored in $-80^{\circ} \mathrm{C}$ for further analysis.

\section{Protein extraction and western immunoblotting}

FC and BR tissues were used for Western immunoblotting analysis to determine the levels of various proteins as described previously [66]. Briefly, tissue samples were sonicated in $0.4 \mathrm{ml}$ of ice-chilled $1 \%$ sodium dodecyl sulphate (SDS) and immediately boiled for 10 minutes. Protein concentrations were determined using Bradford Assay (BioRad, Hercules, CA, USA). Protein extracts were separated by SDSpolyacrylamide electrophoresis (PAGE) in slab gels of $12 \%$ polyacrylamide, and transferred to Hybond-P PVDF membranes (Amersham, Baie d'Urfe, QC, Canada). Membranes were incubated with antibodies against fifteen proteins. These were $\gamma \mathrm{H} 2 \mathrm{AX}$, ELK1, AKT1, MeCP2, (1:1000, Cell Signaling, Danvers, MA, USA), KU70, p 53, BCL2, PCNA, FOXP2, LYNX1, DCX (1:1000, Santa Cruz Biotechnology), MBP (1:500, Millipore, Billerica, MA, USA), and DNMT1, MYT1L, actin (1:1000, Abcam, Cambridge, MA). Due to protein size differences and scarcity of tissue, membranes were re-used and re-probed several times. Antibody binding was revealed by incubation with horseradish peroxidase-conjugated secondary antibodies (Amersham) and ECL Plus immunoblotting detection (Amersham). Chemiluminescence was detected by a FluorChem HD2 System (Cell Biosciences/ ProteinSimple, Santa Clara, CA, USA). Unaltered PVDF membranes were stained with Coomassie Blue (BioRad).

\section{RNA extraction and transcriptome profiling}

Total RNA was isolated using TRIzol Reagent (GE Healthcare Life Sciences, Buckinghamshire, UK). RNA samples were quantified using ultraviolet spectroscopy (NanoDrop, Wilmington, DE) and handed over to the Gene Expression Facility for further transcriptome profiling. The RNA was quantified and found to be of a high quality using a Bioanalyser 2100 (Agilent, CA, USA). RNA labeling and microarray hybridization were performed by the Lethbridge Epigenetics Laboratory Illumina Facility. MouseRef-8 v2.0 Expression BeadChip whole-genome expression arrays (Illumina, CA, USA) were used in this study. Each array on the MouseRef- 8 v2.0 Expression BeadChip targets 25,600 well-annotated RefSeq transcripts, over 19,100 unique genes derived from the National Center for Biotechnology Information Reference Sequence (NCBI) and other sources (Illumina). In total, 40 animals were used, five animals per each treatment (CPP, MMC, PCB) and one control per each of two brain regions.

In brief, each RNA sample was amplified using the Ambion Illumina RNA amplification kit with biotin UTP (Enzo, ON, Canada) labeling. The Ambion Illumina RNA amplification kit uses T7 oligo(dT) primer to generate single-stranded cDNA followed by a second strand synthesis to generate double-stranded cDNA, which is then column purified. In vitro transcription was conducted to synthesize biotin-labeled cRNA using T7 RNA polymerase. The cRNA was column purified and checked for size and yield. cRNA was hybridized using standard Illumina protocols with streptavidinCy3 (Amersham, IL, USA). Arrays were scanned on an Illumina BeadStation and analyzed using BeadStudio (Illumina) as recommended by the manufacturer. Normalization, clustering and significance analysis were done by the Lethbridge Epigenetics Laboratory Illumina Facility as previously described $[67,68]$.

Data were normalized and analyzed using Illumina BeadStudio Software. The false discovery rate (FDR) was controlled using the Benjamini-Hochberg method. The Illumina Custom Model took the FDR into account and was used to analyze the data. Differential gene expression from control cells was determined to be statistically significant if the $p$-value was lower than 0.05 after the Benjamini-Hochberg method adjustment.

\section{Bioinformatics analysis}

\section{Reactome and GO term enrichment analysis}

Pathway information for the differentially expressed genes was retrieved from the Reactome database (http:// www.reactome.org). Pathway enrichment analysis was performed by comparing the number of genes included in the pathways for up- or down-regulated genes, after 
normalizing them against genes in the database. In addition to pathway annotations using the Reactome database, additional annotations with gene ontology (GO) terms were performed using the Database for Annotation, Visualization and Integrated Discovery (DAVID v6.7, http://david.abcc.ncifcrf.gov). GO term enrichments were calculated by dividing the ratio between genes found for a specific term and all genes in the up- or down-regulated set by the ratio between all known mouse genes for a specific term and all known mouse genes:

$\mathrm{E}=\mathrm{N}$ GOt/ Nt: N GOt hg/Nt hg, where $\mathrm{E}=$ enrichment; $\mathrm{N}$ GOt $=$ number of genes assigned to a specific GO term among the up- or down-regulated genes in CPP treatment group; $\mathrm{Nt}=$ total number of up- or downregulated genes in the group; $\mathrm{N}$ GOt hg = number of genes assigned to a specific GO term in the entire mouse genome; $\mathrm{Nt} \mathrm{hg}=$ total number of genes in the mouse genome.

Genes belonging to specific GO terms were than clustered according to DAVID clustering of annotation terms using the Functional Annotation Clustering tool for the following categories: molecular function and biological process.

\section{Gene set analysis (GSA) using KEGG pathways}

The Generally Applicable Gene-set Enrichment (GAGE) method was used as a GSA as previously published [69]. We used the KEGG pathways gene set for comparative analysis. The data for the control group of genes were averaged across five samples and each individual sample in the CPP group was analyzed against the averaged control. The mean log fold change of the genes in the specific KEGG pathway was compared to the mean log fold change of all of the genes on the array. First, a unidirectional analysis for the up- or down-regulated genes was performed, and the up- and down-regulated pathways were identified. Next, a bidirectional analysis was performed, disregarding the direction of the change in gene expression, and the up- or down-regulated pathways were obtained that contained both up- and down-regulated genes. The heat maps were than generated using the FDR adjusted $p$-value of $<0.05$. Specific KEGG pathways show data for the expression of individual genes in all five animals in the CPP group related to the averaged control (Supplementary Figures 1-10).

\section{ACKNOWLEDGMENTS AND FUNDING}

We are grateful to Dr. Igor Kovalchuk for stimulating discussions, critical reading of the manuscript and constructive suggestions. We thank Karen Dow-Kazal and Charlotte Holmes for animal care. This research was supported by the Canadian Institutes of Health Research Grant \#102652 and National Sciences and Engineering Research Council of Canada DG \#298194-20009 (Dr. Gerlinde Metz), and Canadian Institutes for Health Research (Dr. Olga Kovalchuk). Anna Kovalchuk was a recipient of the Canada Vanier Graduate Scholarship and the Alberta Innovates-Health Solutions Graduate Scholarship.

\section{CONFLICTS OF INTEREST}

None.

\section{REFERENCES}

1. Oeffinger KC, Nathan PC, Kremer LC. Challenges after curative treatment for childhood cancer and long-term follow up of survivors. Pediatr Clin North Am. 2008; 55:251-273, xiii.

2. Oeffinger KC, Nathan PC, Kremer LC. Challenges after curative treatment for childhood cancer and long-term follow up of survivors. Hematol Oncol Clin North Am. 2010; 24:129-149.

3. Board AC. Alberta Cancer Board, Cancer in Alberta: A regional picture. 2006.

4. Ries LAG, Eisner MP, Kosary CL, Hankey BF, Miller BA, Clegg L, Mariotto A, Feuer EJ, Edwards BK. editors. SEER cancer statistics review, 1973-1999. Bethesda, MD: National Cancer Institute. 2002.

5. Hewitt M, Weiner SL, Simone JV, editors. Childhood cancer survivorship: improving care and quality of life. Washington (DC): National Academy of Sciences. 2003.

6. Glen CD, Dubrova YE. Exposure to anticancer drugs can result in transgenerational genomic instability in mice. Proc Natl Acad Sci USA. 2012; 109:2984-2988.

7. Glen CD, Smith AG, Dubrova YE. Single-molecule PCR analysis of germ line mutation induction by anticancer drugs in mice. Cancer Res. 2008; 68:3630-3636.

8. Robison LL, Mertens A. Second tumors after treatment of childhood malignancies. Hematol Oncol Clin North Am. 1993; 7:401-415.

9. Kabasenche WP, Skinner MK. DDT, epigenetic harm, and transgenerational environmental justice. Environ Health. 2014; 13:62.

10. Skinner MK. Environmental Epigenetics and a Unified Theory of the Molecular Aspects of Evolution: A NeoLamarckian Concept that Facilitates Neo-Darwinian Evolution. Genome Biol Evol. 2015; 7:1296-1302.

11. Barber RC, Dubrova YE. The offspring of irradiated parents, are they stable? Mutation research. 2006; 598:50-60.

12. Niwa O. Radiation induced dynamic mutations and transgenerational effects. J Radiat Res. 2006; 47:B25-30.

13. Streffer C. Transgenerational transmission of radiation damage: genomic instability and congenital malformation. J Radiat Res. 2006; 47:B19-24.

14. Morgan WF. Non-targeted and delayed effects of exposure to ionizing radiation: II. Radiation-induced genomic instability and bystander effects in vivo, clastogenic factors and transgenerational effects. Radiat Res. 2003; 159:581-596. 
15. Dubrova YE. Radiation-induced transgenerational instability. Oncogene. 2003; 22:7087-7093.

16. Nakamura N, Suyama A, Noda A, Kodama Y. Radiation effects on human heredity. Annu Rev Genet. 2013; 47:33-50.

17. Nomura T. Transgenerational effects of radiation and chemicals in mice and humans. J Radiat Res. 2006; 47:B83-97.

18. Kovalchuk O, Baulch JE. Epigenetic changes and nontargeted radiation effects-is there a link? Environ Mol Mutagen. 2008; 49:16-25.

19. Nomura T. Transgenerational carcinogenesis: induction and transmission of genetic alterations and mechanisms of carcinogenesis. Mutat Res. 2003; 544:425-432.

20. Ryabokon NI, Goncharova RI. Transgenerational accumulation of radiation damage in small mammals chronically exposed to Chernobyl fallout. Radiat Environ Biophys. 2006; 45:167-177.

21. Dasenbrock C, Tillmann T, Ernst H, Behnke W, Kellner R, Hagemann G, Kaever V, Kohler M, Rittinghausen S, Mohr U, Tomatis L. Maternal effects and cancer risk in the progeny of mice exposed to X-rays before conception. Exp Toxicol Pathol. 2005; 56:351-360.

22. Nomura T. X-ray- and chemically induced germ-line mutation causing phenotypical anomalies in mice. Mutat Res. 1988; 198:309-320.

23. Barber RC, Hickenbotham $P$, Hatch $T$, Kelly $D$, Topchiy N, Almeida GM, Jones GD, Johnson GE, Parry JM, Rothkamm K, Dubrova YE. Radiation-induced transgenerational alterations in genome stability and DNA damage. Oncogene. 2006; 25:7336-7342.

24. Koturbash I, Baker M, Loree J, Kutanzi K, Hudson D, Pogribny I, Sedelnikova O, Bonner W, Kovalchuk O. Epigenetic dysregulation underlies radiation-induced transgenerational genome instability in vivo. Int $\mathrm{J}$ Radiat Oncol Biol Phys. 2006; 66:327-330.

25. Tamminga J, Koturbash I, Baker M, Kutanzi K, Kathiria P, Pogribny IP, Sutherland RJ, Kovalchuk O. Paternal cranial irradiation induces distant bystander DNA damage in the germline and leads to epigenetic alterations in the offspring. Cell Cycle. 2008; 7:1238-1245.

26. Nomura T. Transgenerational effects from exposure to environmental toxic substances. Mutat Res. 2008; 659: 185-193.

27. Yauk C, Polyzos A, Rowan-Carroll A, Somers CM, Godschalk RW, Van Schooten FJ, Berndt ML, Pogribny IP, Koturbash I, Williams A, Douglas GR, Kovalchuk O. Germline mutations, DNA damage, and global hypermethylation in mice exposed to particulate air pollution in an urban/ industrial location. Proc Natl Acad Sci USA. 2008; 105:605-610.

28. Merrifield M, Kovalchuk O. Epigenetics in radiation biology: a new research frontier. Front Genet. 2013; 4:40

29. Babenko O, Kovalchuk I, Metz GA. Stress-induced perinatal and transgenerational epigenetic programming of brain development and mental health. Neurosci Biobehav Rev. 2015; 48:70-91.

30. Ward ID, Zucchi FC, Robbins JC, Falkenberg EA, Olson DM, Benzies K, Metz GA. Transgenerational programming of maternal behaviour by prenatal stress. BMC pregnancy and childbirth. 2013; 13:S9.

31. Sedelnikova OA, Pilch DR, Redon C, Bonner WM. Histone $\mathrm{H} 2 \mathrm{AX}$ in DNA damage and repair. Cancer Biol Ther. 2003; 2:233-235.

32. Rufini A, Tucci P, Celardo I, Melino G. Senescence and aging: the critical roles of p53. Oncogene. 2013; 32: 5129-5143.

33. Williams AB, Schumacher B. p53 in the DNA-DamageRepair Process. Cold Spring Harb Perspect Med. 2016; 6.

34. Hoeijmakers JH. Genome maintenance mechanisms for preventing cancer. Nature. 2001; 411:366-374.

35. Hoeijmakers JH. Genome maintenance mechanisms are critical for preventing cancer as well as other aging-associated diseases. Mechanisms of ageing and development. 2007; 128:460-462.

36. Youle RJ, Strasser A. The BCL-2 protein family: opposing activities that mediate cell death. Nat Rev Mol Cell Biol. 2008; 9:47-59.

37. Easton RM, Cho H, Roovers K, Shineman DW, Mizrahi M, Forman MS, Lee VM, Szabolcs M, de Jong R, Oltersdorf T, Ludwig T, Efstratiadis A, Birnbaum MJ. Role for Akt3/ protein kinase Bgamma in attainment of normal brain size. Mol Cell Biol. 2005; 25:1869-1878.

38. Yang ZZ, Tschopp O, Baudry A, Dummler B, Hynx D, Hemmings BA. Physiological functions of protein kinase B/Akt. Biochem Soc Trans. 2004; 32:350-354.

39. Jaenisch R, Bird A. Epigenetic regulation of gene expression: how the genome integrates intrinsic and environmental signals. Nat Genet. 2003; 33:245-254.

40. Nguyen MV, Du F, Felice CA, Shan X, Nigam A, Mandel G, Robinson JK, Ballas N. MeCP2 is critical for maintaining mature neuronal networks and global brain anatomy during late stages of postnatal brain development and in the mature adult brain. J Neurosci. 2012; 32:10021-10034.

41. Guy J, Cheval H, Selfridge J, Bird A. The role of MeCP2 in the brain. Annu Rev Cell Dev Biol. 2011; 27:631-652.

42. Zucchi FC, Yao Y, Ward ID, Ilnytskyy Y, Olson DM, Benzies K, Kovalchuk I, Kovalchuk O, Metz GA. Maternal stress induces epigenetic signatures of psychiatric and neurological diseases in the offspring. PLoS One. 2013; 8:e56967.

43. Wang T, Zeng Z, Li T, Liu J, Li J, Li Y, Zhao Q, Wei Z, Wang Y, Li B, Feng G, He L, Shi Y. Common SNPs in myelin transcription factor 1-like (MYT1L): association with major depressive disorder in the Chinese Han population. PLoS One. 2010; 5:e13662.

44. Stevens SJ, van Ravenswaaij-Arts CM, Janssen JW, Klein Wassink-Ruiter JS, van Essen AJ, Dijkhuizen T, van Rheenen J, Heuts-Vijgen R, Stegmann AP, Smeets EE, Engelen JJ. MYT1L is a candidate gene for intellectual 
disability in patients with 2p25.3 (2pter) deletions. Am J Med Genet A. 2011; 155A:2739-2745.

45. Han H, Myllykoski M, Ruskamo S, Wang C, Kursula P. Myelin-specific proteins: a structurally diverse group of membrane-interacting molecules. Biofactors. 2013; 39: 233-241.

46. Reiriz AB, Reolon GK, Preissler T, Rosado JO, Henriques JA, Roesler R, Schwartsmann G. Cancer chemotherapy and cognitive function in rodent models: memory impairment induced by cyclophosphamide in mice. Clinical cancer research. 2006; 12:5000; author reply 5000-5001.

47. Yang M, Kim JS, Song MS, Kim SH, Kang SS, Bae CS, Kim JC, Wang H, Shin T, Moon C. Cyclophosphamide impairs hippocampus-dependent learning and memory in adult mice: Possible involvement of hippocampal neurogenesis in chemotherapy-induced memory deficits. Neurobiology of learning and memory. 2010; 93:487-494.

48. Christie LA, Acharya MM, Parihar VK, Nguyen A, Martirosian V, Limoli CL. Impaired cognitive function and hippocampal neurogenesis following cancer chemotherapy. Clinical cancer research. 2012; 18:1954-1965.

49. Hsu LL, Adams PM, Legator MS. Cyclophosphamide: effects of paternal exposure on the brain chemistry of the F1 progeny. Journal of toxicology and environmental health. 1987; 21:471-481.

50. Adams PM, Fabricant JD, Legator MS. Cyclophosphamideinduced spermatogenic effects detected in the F1 generation by behavioral testing. Science. 1981; 211:80-82.

51. Adams PM, Fabricant JD, Legator MS. Active avoidance behavior in the F1 progeny of male rats exposed to cyclophosphamide prior to fertilization. Neurobehavioral toxicology and teratology. 1982; 4:531-534.

52. Lyons L, Elbeltagy M, Bennett G, Wigmore P. The effects of cyclophosphamide on hippocampal cell proliferation and spatial working memory in rat. PloS one. 2011; 6:e21445.

53. Feng Z, Li K, Liu M, Wen C. NRAGE is a negative regulator of nerve growth factor-stimulated neurite outgrowth in PC12 cells mediated through TrkA-ERK signaling. Journal of neuroscience research. 2010; 88:1822-1828.

54. Gao HL, Xu H, Xin N, Zheng W, Chi ZH, Wang ZY. Disruption of the CaMKII/CREB signaling is associated with zinc deficiency-induced learning and memory impairments. Neurotoxicity research. 2011; 19:584-591.

55. Klempin F, Kronenberg G, Cheung G, Kettenmann H, Kempermann G. Properties of doublecortin-(DCX)-expressing cells in the piriform cortex compared to the neurogenic dentate gyrus of adult mice. PloS one. 2011; 6:e25760.

56. Ocklenburg S, Arning L, Gerding WM, Epplen JT, Gunturkun O, Beste C. FOXP2 variation modulates functional hemispheric asymmetries for speech perception. Brain and language. 2013; 126:279-284.

57. Anglada-Huguet M, Giralt A, Perez-Navarro E, Alberch J, Xifro X. Activation of Elk-1 participates as a neuroprotective compensatory mechanism in models of Huntington's disease. Journal of neurochemistry. 2012; 121:639-648.

58. Buzdin AA, Prassolov V, Zhavoronkov AA, Borisov NM. Bioinformatics Meets Biomedicine: OncoFinder, a Quantitative Approach for Interrogating Molecular Pathways Using Gene Expression Data. Methods Mol Biol. 2017; 1613:53-83.

59. Ozerov IV, Lezhnina KV, Izumchenko E, Artemov AV, Medintsev S, Vanhaelen Q, Aliper A, Vijg J, Osipov AN, Labat I, West MD, Buzdin A, Cantor CR, et al. In silico Pathway Activation Network Decomposition Analysis (iPANDA) as a method for biomarker development. Nat Commun. 2016; 7:13427.

60. Artcibasova AV, Korzinkin MB, Sorokin MI, Shegay PV, Zhavoronkov AA, Gaifullin N, Alekseev BY, Vorobyev NV, Kuzmin DV, Kaprin AD, Borisov NM, Buzdin AA. MiRImpact, a new bioinformatic method using complete microRNA expression profiles to assess their overall influence on the activity of intracellular molecular pathways. Cell Cycle. 2016; 15:689-698.

61. Flurkey K, Yuan R. How the evolutionary theory of aging can guide us in the search for aging genes. Aging (Albany NY). 2012; 4:318-319.https://doi.org/10.18632/aging.100460.

62. Deluty JA, Atzmon G, Crandall J, Barzilai N, Milman S. The influence of gender on inheritance of exceptional longevity. Aging (Albany NY). 2015; 7:412-418.https:// doi.org/10.18632/aging.100763.

63. Baulch JE, Raabe OG, Wiley LM. Heritable effects of paternal irradiation in mice on signaling protein kinase activities in F3 offspring. Mutagenesis. 2001; 16:17-23.

64. Barber RC, Hardwick RJ, Shanks ME, Glen CD, Mughal SK, Voutounou M, Dubrova YE. The effects of in utero irradiation on mutation induction and transgenerational instability in mice. Mutat Res. 2009; 664:6-12.

65. Pletsa V, Valavanis C, van Delft JH, Steenwinkel MJ, Kyrtopoulos SA. DNA damage and mutagenesis induced by procarbazine in lambda lacZ transgenic mice: evidence that bone marrow mutations do not arise primarily through miscoding by O6-methylguanine. Carcinogenesis. 1997; 18:2191-2196.

66. Silasi G, Diaz-Heijtz R, Besplug J, Rodriguez-Juarez R, Titov V, Kolb B, Kovalchuk O. Selective brain responses to acute and chronic low-dose X-ray irradiation in males and females. Biochem Biophys Res Commun. 2004; 325:1223-1235.

67. Luzhna L, Kovalchuk O. Low dose irradiation profoundly affects transcriptome and microRNAme in rat mammary gland tissues. Oncoscience. 2014; 1:751-762. https://doi. org/10.18632/oncoscience.94.

68. Sidler C, Woycicki R, Li D, Wang B, Kovalchuk I, Kovalchuk O. A role for SUV39H1-mediated H3K9 trimethylation in the control of genome stability and senescence in WI38 human diploid lung fibroblasts. Aging (Albany NY). 2014; 6:545-563. https://doi.org/10.18632/aging.100678.

69. Luo W, Friedman MS, Shedden K, Hankenson KD, Woolf PJ. GAGE: generally applicable gene set enrichment for pathway analysis. BMC Bioinformatics. 2009; 10:161. 